\title{
Advances in (and a Brief History of) Cathodoluminescence Microscopy
}

\author{
DJ Stowe ${ }^{1}$, M. Bertilson ${ }^{2}$ and JA. Hunt ${ }^{2}$ \\ 1. Gatan Inc., 25 Nuffield Way, Abingdon, Oxon., UK. \\ 2. Gatan Inc., 5794 W Las Positas Blvd, Pleasanton, USA.
}

Cathodoluminescence (CL) microscopy utilizes the emission of light from a material upon interaction with an electron. Luminescence in the ranges from ultraviolet to infrared wavelengths and may be analysed spatially, spectrally and temporally to form images, spectra and spectrum-images (where each pixel in a data set contains a full spectrum). CL performed in a scanning or scanning transmission electron microscope (SEM or STEM) benefits from the fact that, in these instruments, an electron beam can be made arbitrarily small compared to the (often) nanometer scale mechanism(s) driving the optical behaviours of materials. Furthermore, direct correlation with morphology, microstructure, composition and chemistry using other analytical techniques available in the electron microscope make CL a very attractive characterization tool. Here we will review the astonishing progress made recently using the CL technique from quantification of trace impurities, revealing the local density of optical states in metallic nanoparticles far below the optical diffraction limit to the study of individual quantum emitters and look forward to the exciting research possibilities in the near future.

The phenomena of cathodoluminescence was first reported in 1879 by Crooks who examined the bluegreen light emitted from synthetic calcium sulfide crystals bombarded with a cathode ray [1]. However, it wasn't until the advent of its use in petrographic studies in the 1960s and 1970s that CL found widespread application where CL imaging and spectroscopy were used for the reconstruction of processes of mineral formation and alteration by the visualization of growth textures. Since the mid-1980s, panchromatic CL imaging (collecting and detecting luminescence without recourse to dispersion or filtering) has been widely used to visualize $\mathrm{U} / \mathrm{Pb}$ segregation and zonation within zircon grains for accurate isotope concentration measurements made using ion probe measurements for geochronology [2]. More recently, quantification of titanium trace element concentrations below $100 \mathrm{ppm}$ in quartz has been enabled by color CL and spectrum-imaging approaches for thermobarometry purposes [3].

With the growth in the optoelectronics market, spectrally resolved CL gained great popularity. The most favored and successful approach involves direct optical coupling between a specimen, a monochromator, and finally a photo-detector (photomultiplier tube or charge coupled device). CL has been used extensively to analyse point and extended defects in bulk compound semiconductors and nanostructures e.g. [4] and [5] and recently to elucidate the local density of optical states in metallic nanoparticles with analysis modalities now including angularly resolved CL [6]. The strong interest in developing light emitting diodes emitting at deep ultraviolet wavelengths $(<250 \mathrm{~nm})$ for water purification applications and the expansion in narrow bandgap semiconductors for mid- and long-wavelength infrared detectors $(3-10$ $\mu \mathrm{m}$ ) has seen the development of spectrally resolved CL systems free from chromatic aberration, expanding the range of materials that can be analysed successfully using CL [7].

The explosion in nanotechnology applications in the last 15 years has driven the expansion of the CL technique with new applications and analytical modalities being developed as the limitations of optical spectroscopy techniques such as electro- and photo-luminescence are restricted in their ability to study objects below the far field diffraction limit (i.e. $<200 \mathrm{~nm}$ ). The advent of SEMs suitable for operation 
with small probe sizes at low accelerating voltages together with the development of optimized detection hardware has pushed spatial resolution of CL measurements in bulk specimens to a few tens of nanometers and in the case of thin specimens (analysed in the STEM), into a regime where the sample's energy transfer processes effectively defines the spatial resolution. However, as most (semiconductor) objects of interest are efficient charge-carrier traps (e.g. quantum dots, discs and wells) the diffusion length (and thus the spatial resolution) are essentially determined by the size of the object of interest [9] and [10]; an example is shown in Figure 1.

Here we present how this very recent and unprecedented access to material's optical and electronic properties at the length scale of critical device features is being used to advance our understanding of technologically important materials including bandgap analysis at the nanoscale [10], development of LED structures with higher efficiencies due to mitigation of the quantum confined Stark effect [11] and development of (solid state) quantum dots [9].

\section{References:}

[1] W Crookes, Phil Trans Royal Soc London 170 (1897) p. 1495.

[2] IS Williams et al., Contrib Mineral Petrol 88 (1984) p. 32.

[3] WP Leeman et al., Microsc Microanal. 18(6) (2012) p. 1322.

[4] MR Phillips, O Gelhausen and EM Goldys, Phys. Stat. Sol. A 201(2) (2004) p. 229.

[5] E Dupuy et al., Appl Phys Letts 94 (2009) p. 022113.

[6] T Coenen et al., Nano Lett. 11 (2011) p. 3379.

[7] OC Noriega et al., US Workshop on the Physics and Chemistry of II-VI Materials (2012)

[9] LF Zagonel et al., Nano Lett. 11 (2011) p. 568.

[10] GR Fern, J Silver and S Coe-Sullivan, J. Soc. Information Display 23 (2) (2015) p. 50.

[11] JT Griffiths et al., Nano Lett. 15 (2015) p. 7639.

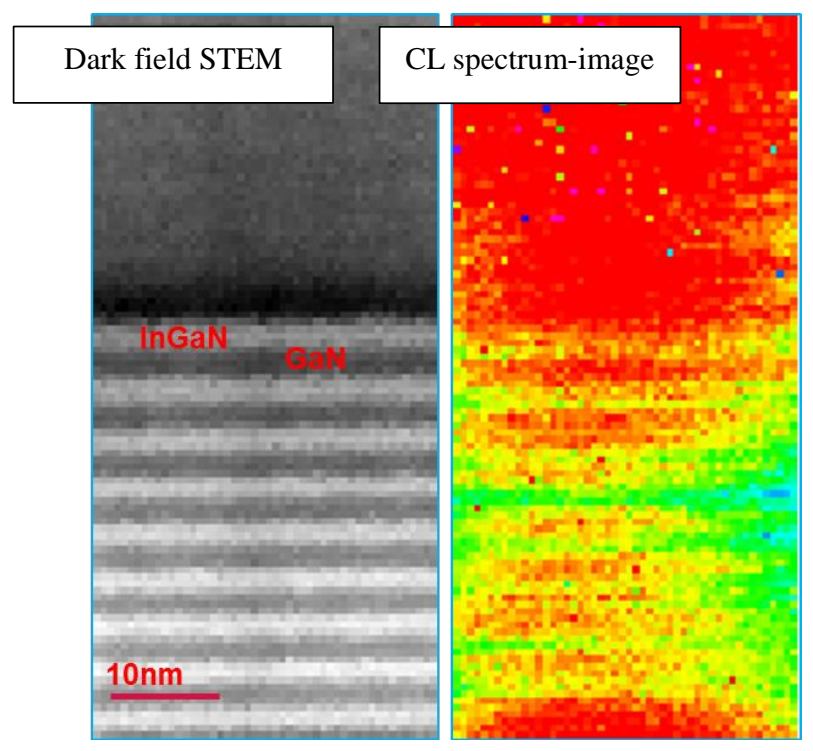

Figure 1. Optoelectronic properties revealed by CL spectrum-imaging with a spatial resolution at the feature length of individual InGaN quantum wells in a commercially available LED. Efficient chargecarrier capture provides a CL spatial resolution defined by the size of the object of interest. 